\title{
Empirical Evidence for the Relationship Between Cognitive Workload and Attentional Reserve
}

Kyle J. Jaquess ${ }^{1}$, Li-Chuan Lo ${ }^{1}$, Hyuk $\mathrm{Oh}^{1,2}$, Clayton A. Domingues, ${ }^{1,3,4}$, Seth R. Neiman ${ }^{1,5}$, Jeremy C. Rietschel ${ }^{6}$, Matthew W. Miller ${ }^{7}$, Ying Y. Tan ${ }^{1,2}$, Rodolphe J. Gentili ${ }^{1,2,8}$, \& Bradley D. Hatfield ${ }^{1,2}$. ${ }^{1}$ Department of Kinesiology, University of Maryland, College Park, MD, ${ }^{2}$ Neuroscience And Cognitive Science program, University of Maryland, College Park, MD, ${ }^{3}$ Department of Neurology, Federal Fluminense University, Niterói, Brazil, ${ }^{4}$ Special Operations Instruction Center, Niterói, Brazil, ${ }^{5}$ Center for Enhanced Performance, United States Military Academy, West Point, New York, ${ }^{6}$ Veterans Health Administration, Maryland Exercise and Robotics Center of Excellence, Baltimore, MD, ${ }^{7}$ School of Kinesiology, Auburn University, Auburn, AL, ${ }^{8}$ Maryland Robotics Center, University of Maryland, College Park, MD

In an environment with increasingly complex and challenging situations, there is an evergrowing requirement to manage the demand on one's mental systems in an adaptive manner so as to maximize productivity and performance. The capacity of the cognitive system can be divided into two basic elements: cognitive workload (CW), or the processes concerning taskrelevant information, and attentional reserve (AR), the portion of the capacity that it unused by the task. Theoretically, these two constructs have an inverse relationship, but this has never been explicitly tested. PURPOSE: The present experiment focused on using electroencephalography (EEG) and event-related potentials (ERPs) as measures of CW and AR, respectively (Gentili et al., Submitted; Miller, Rietschel, McDonald, \& Hatfield, 2011) in an effort to provide empirical data to support or refute this theoretical prediction. It is hypothesized that as EEG measures of CW increase, ERP measures of AR will decrease. METHODS: Participants (27 males) performed a flight simulation task under three levels of nominal difficulty (low, medium, high) while EEG spectral measures of cortical activation and ERP data were collected. Canonical correlations were conducted on difference scores between each condition to test the statistical magnitude and significance of the relationship between EEG and ERP measures. RESULTS: Results from a canonical correlation (cancorr) analysis revealed the predicted result; collectively, measures of CW (spectral measures of cortical activation) have a strong inverse relationship to measures of AR (ERP components) (Low - Medium: Cancorr Coefficient $=-0.783, \mathrm{p}=0.047$; Low - High: Cancorr Coefficient: $-0.791, p=0.038$; Medium - High: Cancorr Coefficient $=$ 0.817, $\mathrm{p}=0.019$ ). CONCLUSION: These findings support the present hypothesis; as measures believed to represent $\mathrm{CW}$ increase, measures believed to represent AR decrease. Future work must be conducted to assess reference values, or "anchor points", for these measures of CW and AR to understand their practical implications (i.e., How high/low can measures of CW/AR be?). Until such information is gathered, it will not be possible to understand the magnitude of the changes observed in physiological measures of CW and AR. Supported by the Lockheed Martin Corporation. 